\title{
AVALIAÇÃO DA QUALIDADE DA INFORMAÇÃO SOBRE A CAUSA BÁSICA DE ÓBITOS INFANTIS NO RIO GRANDE DO SUL (BRASIL)*
}

\author{
Letícia C. Nobre** \\ Cesar G. Victora** \\ Fernando C. Barros** \\ Cintia Lombardi** \\ Ana Maria B. Teixeira** \\ Sandra C. Fuchs**
}

\begin{abstract}
NOBRE, L.C. et al. Avaliaçăo da qualidade da informaçăo sobre a causa básica de óbitos infantis no Rio Grande do Sul (Brasil). Rev. Saúde públ., S. Paulo, 23: 207-13, 1989.

RESUMO: A partir de dados coletados para um estudo de casos e controles sobre mortalidade infantil por doenças infecciosas e desnutriçăo realizado em Porto Alegre e Pelotas, RS (Brasil), comparou-se as causas de obito constantes do atestado médico com as causas obtidas através de uma revisão detalhada, realizada pela equipe da investigação. Concluiụ-se que as estatísticas oficiais năo são fidedignas, tendo havido sobre-registro de broncopneumonias (CID 485X) e de septicemias (CID 038.9), e sub-registro de gastroenterites (CID 009.1) e de mortes súbitas (CID 798.0). A concordância entre os atestados refeitos e os oficiais, em termos de grupos de causas de óbito, foi de apenas $27.9 \%$.
\end{abstract}

DESCRITORES: Mortalidade infantil. Classificaçăo de doenças. Atestados de b́bitos

\section{INTRODUÇÃO}

O coeficiente de mortalidade infantil tem sido amplamente utilizado como indicador de saúde e de qualidade de vida das populações 5 . No entanto, esse indicador é freqüentemente afetado pelo sub-registro de nascimentos e óbitos, o que faz com que as estatísticas vitais em diversas regióes do Brasil não sejam fidedignas'. Mesmo para os óbitos registrados existe o problema da qualidade da informação sobre a causa básica. Por exemplo, durante a "Investigação Interamericana de Mortalidade na Infância", a comparação entre os atestados de óbito oficiais e os atestados revisados pela equipe do estudo revelou um índice de concordância global de apenas $46,3 \%$.

Para algumas causas de óbito, particularmente aquelas que aparecem freqüentemente como complicações de outras patologias, pode ocorrer o problema da sobre-enumeração. Por exemplo, Fonseca e Laurenti ${ }^{3}$ e Laurenti ${ }^{4}$ mostraram em estudo realizado em São Paulo que as pneumonias eram sempre sobre-enumeradas como causas de óbito. Também no estudo " $\mathrm{Ca}$ racterísticas da Mortalidade Urbana" ", realizado na década de 1960 , no Município de São Paulo, verificou-se um sobre-registro de $35 \%$ de pneumonias como causa básica de óbito.

No Estado do Rio Grande do Sul têm sido descritas reduções sucessivas nos coeficientes de mortalidade infantil nos últimos anos, com diminuição dos óbitos por enterites e gastroenterites e aumento relativo das infecções respiratórias, principalmente broncopneumonias, que passaram a ser as causas infecciosas mais importantes de mortalidade em menores de um ano $^{10}$.

Para melhor estimar a magnitude das causas de óbitos infantis naquele Estado, realizou-se uma comparação entre os dados oficiais e uma revisão pormenorizada da patologia que levou à morte. Este tipo de informação é de importância pois, pelo conhecimento da magnitude das diferentes causas infecciosas de mortalidade infantil, pode-se chegar a estratégias mais efetivas de prevenção e controle.

\section{METODOLOGIA}

A partir de dados coletados para estudo de casos e controles sobre mortalidade infantil, comparou-se as causas de óbito constantes do atestado de óbito com as causas obtidas por meio de revisão pormenorizada pela equipe de investigadores. $\mathrm{O}$ objetivo da investigação foi cobrir todos os casos de óbitos de crianças entre 7 e 364 dias de idade, cuja causa básica fosse alguma doença infecciosa ou desnutrição. O estudo foi restrito a óbitos de crianças residentes nas áreas urbanas da Região Metropolitana de

" Estudo financiado pelo "International Development Research Centre do Canadá". Processo 3-P-84-0200/9

* Departamento de Medicina Social da Faculdade de Medicina da Universidade Federal de Pelotas - Caixa Potal 464 96001 - Pelotas, RS - Brasil. 
Porto Alegre e da cidade de Pelotas, ocorridos no período compreendido entre 24 de dezembro de 1984 e 23 de dezembro de 1985. A metodologia empregada encontra-se descrita em outras publicaçð̃es ${ }^{11,12}$.

Para identificação dos óbitos, foram visitados semanalmente todos os hospitais, Institutos de Medicina Legal (IMLs), Delegacias da Secretaría de Saúde e Meio Ambiente (SSMA) e Cartórios de Registros localizados na área em estudo. Em Pelotas, foi também necessário visitar os cemitérios locais, pois havia indicação da existência de sub-registro importantel. Os atestados de óbito foram revisados semanalmente junto à Unidade de Informática da SSMA e conferidos com os casos identificados através das outras fontes. Além disso, foi feita revisão final, no início de 1986, para deteç̧ão dos atestados nã̃o localizados durante o transcorrer do ano.

Do total dos óbitos ocorridos, foram excluidas as crianças portadoras de malformaçðes congênitas, de peso de nascimento abaixo de $1500 \mathrm{~g}$ e aquelas que permaneceram em berçário por mais de 15 dias após o nascimento. Estas exclusões foram necessárias devido ao desenho do estudo de casos e controles ${ }^{12}$.

Sempre que uma doença infecciosa ou por desnutrição tivesse sido declarada como causa básica ou associada de óbito, ou quando a causa era mal definida ou não declarada, um médico realizava visita domiciliar aos pais da criança falecida para investigar a causa do óbito. Foi possível, através de várias tentativas, localizar $96,5 \%$ deles. Durante as visitas, o médico coletava informaçōes sobre o episódio da doença que levou ao óbito e sobre os antecedentes mórbidos. Foram também revisados os prontuários das internações hospitalares, os laudos de necrópsia e os registros de postos de saúde, quando disponíveis. Se necessário, realizava-se uma entrevista com o médico que havia assistido a criança. Para a coleta desses dados foram utilizados os mesmos formulários aplicados na "Investigação Interamericana de Mortalidade na Infância"'s.

Dois médicos avaliaram, separadamente, as informações assim coletadas e refizeram o atestado de óbito, baseados na IX Revisão da Classificação Internacional de Doenças ${ }^{6}$. Em caso de discordância entre os investigadores, um dos coordenadores da pesquisa tomava a decisão final. Os atestados assim refeitos foram comparados com os atestados oficiais, fornecidos pela SSMA.

Para mortes cujas causas não tivessem sido suficientemente explicadas, utilizou-se a classificação "mal-definida" ou "morte súbita de causa desconhecida", sendo: a) Mal-definida (CID 799.9): mortes mal explicadas acompanhadas de sintomas leves ou vagos que ocorreram mais de 24 horas após o início dos sintomas;

b) Morte súbita de causa desconhecida (CID 798.0): morte repentina com criança assintomática até 24 horas antes de sua ocorrência.

Para fins do presente estudo, a desnutrição (CID 263.9) foi considerada como causa básica nos casos em que a causa inicialmente determinada pelos revisores era broncopneumonia (CID 485X), pneumonia (CID 482.9) ou gastroenterite (CID 009.3) e em que havia desnutrição grave associada. Considerou-se como critério de desnutrição grave um peso, por ocasião da internação que levou ao óbito, três ou mais desvios-padrão abaixo da mediana da referência do "National Center for Health Statistics"? de peso por idade. Cabe notar que em $46.5 \%$ dos óbitos não havia registro de peso por ocasião da internação.

Para fins de comparação, as causas de óbito foram divididas nos seguintes grupos: gastroenterites (CID 003.0; 004.1; 008.0; 009.0; 009.1; 009.3), bronquiolite (CID 466.1), pneumonias (CID $480.9 ; 481.0 ; 482.9 ; 486 \mathrm{X}$ ), broncopneumonias (CID 485X), outras infecções respiratórias (CID 382.0; 382.9; 464.0;465.9; 487.0; 487.1; 519.8), doenças infecciosas específicas (CID 011.9; 013.0; 018.9; 033.9; 036.2; 052.0; $054.2 ; 054.3 ; 055.1 ; 055.9 ; 070.9 ; 090.9$ ), meningites (CID 320.0; 320.1; 320.5; 320.7; 320.8; 322.9), infecções de pele (CID 682.0; 682.1; $682.5 ; 682.8 ; 684 X ; 686.9$ ), outras infecções (CID 038.9; 136.9; 422.9; 771.2; 771.4; 771.8), desnutrição (CID 260X; 261X; 262X; 263.9), doenças não infecciosas (CID 276.2; 276.9; $277.0 ; 281.9 ; 285.9 ; 323.5 ; 323.9 ; 348.2$; $425.4 ; 428.1 ; 428.9 ; 454.2 ; 478.7 ; 493.9 ; 507.0$; $516.8 ; 518.4 ; 518.8 ; 519.9 ; 530.1 ; 550.1 ; 558.0$; $695.8 ; 745.4 ; 746.0 ; 749.0 ; 770.1 ; 770.8 ; 774.7$; $775.5 ; 780.3 ; 785.5$ ), morte súbita (CID 798.0; 798.9), e causas mal-definidas (CID 799.1; 799.4; 799.9). Esses grupos de causas de óbito foram utilizados no cálculo dos índices de concordância descritos a seguir.

Em vista da metodologia utilizada, consideramos serem os atestados refeitos pelos investigadores mais fidedignos que os oficiais, passando aqueles, portanto, a consistirem no "padrão-ouro" em relação ao qual os atestados oficiais foram julgados. Duas medidas de associação foram utilizadas na análise, a sensibilidade (por exemplo, percentual de óbitos por gastroenterites identificados corretamente no atestado) e o indice kappa para concordância inter-observadores. Este último índice varia de 
+ 1 (concordância total) a - 1 (discordância total). Índices próximos a zero indicam que a concordância entre os dois observadores não foi maior do que aquela que seria esperada simplesmente em função do acaso?.

Comparando-se o número oficial de óbitos por determinado grupo de causas e o número correspondente de óbitos obtidos pela revisão, obteve-se os percentuais de sub-registro ou de sobre-registro, segundo as fórmulas abaixo:

Percentual de sub-registro $=$

$100-\frac{\text { N. oficial }}{\text { N. revisto }} \times 100$

Percentual de sobre-registro $=$

$\frac{\text { N. bficial }}{N^{\circ} \text { revisto }} \times 100 \cdot 100$

\section{RESULTADOS}

Foram estudados 486 óbitos infantis, sendo que em 27,9\% (129 casos) houve concordância entre revisores e atestados oficiais em termos dos grupos de causas descritos acima.

A Tabela 1 mostra que foram oficialmente registrados 107 casos de óbito por gastroenterites, enquanto que o total encontrado pelos revisores foi de 149. Dos 107 casos registrados, 63 eram efetivamente de gastroenterite e 44 casos foram registrados erroneamente. Deixou-se de registrar 86 casos de gastroenterite, sendo o índice de sub-registro nos dados oficiais igual a
$28 \%$ (42 casos). A sensibilidade foi de apenas $42 \%$, e estatística kappa foi igual a 0,32 .

A mesma tabela mostra que dos 44 óbitos por broncopneumonia, 22 constam oficialmente. Por outro lado, 124 casos foram registrados erroneamente, havendo um sobre-registro de $232 \%$, sendo a estatística kappa igual a 0,11 (um valor muito baixo) e a sensibilidade de $50 \%$.

De 84 óbitos identificados pelos revisores como devidos a outras infecções respiratórias (bronquiolites, pneumonias e outras) que não as broncopneumonias, em apenas cinco a causa básica registrada estava correta. Os kappas variaram entre 0 e 0,13 , e a sensibilidade foi de $8 \%$ para bronquiolites, $13 \%$ para pneumonias e $0 \%$ para as restantes infecçōes respiratórias.

Para as demais infecções (doenças infecciosas específicas, meningites, infecções de pele e outras) a sensibilidade foi de $38 \%$, havendo um sub-registro de $44 \%$ para infecções específicas e de $70 \%$ para infecções de pele, e um sobreregistro de $25 \%$ para meningites. Os 12 casos desse grupo diagnóstico que deixaram de ser detectados incluem quatro óbitos por sarampo, dois por infecções herpéticas, um caso de sífilis congênita e outro de hepatite viral.

A Tabela 2 mostra que dos 86 casos de gastroenterite não registrados como tal, 20 o foram como broncopneumonia, 18 como desnutrição, 13 como septicemia, cinco como pneumonia e 14 casos foram registrados como outras causas. Em outros 16 casos, o atestado não foi localizado na SSMA.

TABELA 1

Concordância entre os atestados oficiais de óbitosinfantis e os atestados obtidos após a revisão pela equipe dainvestigação, região Metropolitana de Porto Alegre e Pelotas, RS, 1985.

\begin{tabular}{|c|c|c|c|c|c|c|}
\hline Causa básica & $\begin{array}{l}\text { Número de } \\
\text { óbitos segundo } \\
\text { os revisores }\end{array}$ & $\begin{array}{l}\text { Número } \\
\text { oficial } \\
\text { de óbitos }\end{array}$ & $\begin{array}{l}\text { Obitos em } \\
\text { que houve } \\
\text { concordância }\end{array}$ & $\begin{array}{l}\text { Sensibi- } \\
\text { lidade } \\
(\%)\end{array}$ & Kappa & $\begin{array}{c}\text { Subregis- } \\
\text { tro } \\
(\%)\end{array}$ \\
\hline Gastroenterite & 149 & 107 & 63 & 42 & 0,32 & 28 \\
\hline Bronquiolite & 38 & 4 & 3 & 8 & 0,13 & 89 \\
\hline Pneumonia & 15 & 16 & 2 & 13 & 0,10 & -7 \\
\hline Broncopneumonia & 44 & 146 & 22 & 50 & 0,11 & -232 \\
\hline Outras infecçōes respiratórias & 31 & 5 & 0 & 0 & 0,00 & 84 \\
\hline Infecçōes específicas & 27 & 15 & 12 & 44 & 0,55 & 44 \\
\hline Meningites & 12 & 15 & 9 & 75 & 0,66 & -25 \\
\hline Infeç̧ōes de pele & 10 & 3 & 2 & 20 & 0,30 & 70 \\
\hline Outras infecçöes & 15 & 40 & 1 & 7 & 0,00 & -167 \\
\hline Desnutrição & 40 & 50 & 13 & 33 & 0,22 & -25 \\
\hline Doenças não infecciosas & 13 & 38 & 1 & 8 & 0,00 & -192 \\
\hline Morte súbita & 72 & 5 & 1 & 1 & 0,01 & 93 \\
\hline Mal definidas & 20 & 2 & 0 & 0 & 0,00 & 90 \\
\hline Sem registro & 0 & 40 & - & - & - & - \\
\hline Total & 486 & 486 & 129 & & & \\
\hline
\end{tabular}

Obs.: (a) Percentuais nega tivos de subregistro indicam a existência de sobre-registro. 


\section{TABELA 2}

Causas básicas segundo os atestados oficiais dos óbitos atribuídos a gastroenterites após a revisão. Região Metropolitana de Porto Alegre e Pelotas, RS, 1985.

\begin{tabular}{lrr}
\hline $\begin{array}{l}\text { Causas básicas segundo } \\
\text { os atestados oficiais }\end{array}$ & Número & $\%$ \\
\hline Gastroenterite & 63 & 42 \\
Pneumonia, broncopneumonia & 25 & 17 \\
Desnutrição & 18 & 12 \\
Sepsis & 13 & 9 \\
Outras infecções & 5 & 3 \\
Não infeciosas & 6 & 4 \\
Morte súbita & 2 & 1 \\
Mal-definidas & 1 & 1 \\
Sem registro & 16 & 11 \\
\hline Total & 149 & 100 \\
\hline
\end{tabular}

As Tabelas 3 e 4 mostram como foram certificados os óbitos por broncopneumonia e desnutrição.

\section{TABELA 3}

Causas básicas segundo os atestados oficiais dos óbitos atribuídos a broncopneumonias após a revisāo. Regiāo Metropolitana de Porto Alegre e Pelotas, RS, 1985.

\begin{tabular}{lcr}
\hline $\begin{array}{l}\text { Causas básicas segundo } \\
\text { os atestados oficiais }\end{array}$ & Número & $\%$ \\
\hline Broncopneumonias & 22 & 50 \\
Outras infecçōes & & \\
$\quad$ respira tórias & 3 & 7 \\
Desnutrição & 5 & 11 \\
Sepsis & 3 & 7 \\
Gastroenterite & 3 & 7 \\
Meningite & 1 & 2 \\
Não infecciosas & 3 & 7 \\
Sem registro & 4 & 9 \\
\hline Total & 44 & 100 \\
\hline
\end{tabular}

\section{TABELA 4}

Causas básicas segundo os atestados oficiais dos óbitos atribuídos a desnutrição após a revisão. Regiāo Metropolitana de Porto Alegue e Pelotas, RS, 1985.

\begin{tabular}{lrr}
\hline $\begin{array}{l}\text { Causas básicas segundo } \\
\text { os atestados oficiais }\end{array}$ & Número & $\%$ \\
\hline Desnutrição & 13 & 33 \\
Septicemia & 9 & 23 \\
Broncopneumonia & 8 & 20 \\
Gastroenterites & 7 & 18 \\
Não infecciosa & 1 & 3 \\
Sem registro & 2 & 5 \\
\hline Total & 40 & 102 \\
\hline
\end{tabular}

São chamativos os resultados da Tabela 5 , onde se pode observar que nenhum dos casos de morte súbita foi identificado como tal. A maioria destes $(61 \%)$ foram registrados como devidos a broncopneumonias. Estes resultados já haviam sido apresentados previamente ${ }^{11}$.

\section{TABELA 5}

Causas básicas segundo os atestados oficiais das mortes súbitas na infância. Regiảo Metropolitana de Porto Alegre e Pelotas, RS, 1985.

\begin{tabular}{lrr}
\hline $\begin{array}{l}\text { Causas básicas segundo } \\
\text { os atestados oficiais }\end{array}$ & Número & $\%$ \\
\hline Broncopneum onia & 44 & 61 \\
Gastroenterites & 8 & 11 \\
Infecçöes neonatais & 8 & 11 \\
Outras causas & 5 & 7 \\
Sem registro & 7 & 10 \\
\hline Total & 72 & 100 \\
\hline
\end{tabular}

Outro lado que chama a atenção é o alto número de óbitos atribuídos a septicemia, quando se sabe que essa é na maior parte das vezes um estágio terminal de outras infecções. A Tabela 6 indica que, dos 38 casos registrados como septicemia, cerca de um terço eram devidos a gastroenterites, nove a desnutrição, oito a infecções respiratórias, sendo os restantes devido a outras infecções ou mesmo a doenças não infecciosas.

\section{TABELA 6}

Causas básicas segundo os revisores dos óbitos atribuídos a septicemia pelos atestados oficiajs. Região Metropolitana de Porto Alegre e Pelotas, RS, 1985.

\begin{tabular}{lrr}
\hline $\begin{array}{l}\text { Causas básicas segundo } \\
\text { os revisores }\end{array}$ & Número & $\%$ \\
\hline Gastroenterite & 13 & 34 \\
Desnutrição & 9 & 24 \\
Broncopneumonia & 3 & 8 \\
Bronquiolite & 3 & 8 \\
Infecção de pele & 2 & 5 \\
Outras infeç̧ōes & 2 & 5 \\
respiratórias & 1 & 3 \\
Meningite & 1 & 3 \\
Coqueluche & 1 & 3 \\
Outras infecçōes & 3 & 8 \\
Não infecciosas & 38 & 101 \\
\hline Total & & \\
\hline
\end{tabular}

Na Tabela 7 estão listadas as causas de 40 óbitos $(8,2 \%)$ que não foram registrados na SSMA. Desses, mais de um terço eram devido a gastroenterites. $O$ sub-registro foi extremamien- 
te alto na cidade de Pelotas, onde 29 dos 64 óbitos estudados $(45 \%)$ não chegaram a ser registrados, enquanto que apenas $11(3 \%)$ dos 422 óbitos estudados na Região Metropolitana de Porto Alegre não alcançaram a SSMA.

\section{TABELA 7}

Causas básicas segundo os revisores dos óbitos cujos atestados não chegaram à Secretaria da Saúde. Região Metropolitana de Porto Alegre e Pelotas, RS, 1985.

\begin{tabular}{lrr}
\hline $\begin{array}{l}\text { Causas básicas segundo } \\
\text { os revisores }\end{array}$ & Número & $\%$ \\
\hline Gastroenterite & 16 & 40 \\
Morte súbita & 7 & 18 \\
Broncopneumonia & 4 & 10 \\
Bronquiolite & 3 & 8 \\
Mal-definidas & 3 & 8 \\
Desnutrição & 2 & 5 \\
Outras infeç̄óes & 2 & 5 \\
respiratórias & 1 & 3 \\
Meningite & 2 & 5 \\
Outras infecçōes & 40 & 102 \\
\hline Total & & \\
\hline
\end{tabular}

Em termos ideais, o diagnóstico fornecido por uma necrópsia detalhada, incluindo exame microscópico, apresenta maior grau de certeza do que o diagnóstico clínico e laboratorial. Em nosso meio, no entanto, as necrópsias são realizadas primariamente com objetivos médicolegais, mais precisamente para excluir a possibilidade de morte violenta. Por esses motivos, valorizamos na presente investigação apenas aqueles diagnósticos de necrópsia que eram acompanhados de laudo anátomo-patológico. Para os demais casos - que constituíram a grande maioria - os dados sobre a causa básica foram obtidos pelas entrevistas com os pais e prontuários médicos, como descrito acima.

A Tabela 8 mostra a comparação entre as causas básicas declaradas pelos IMLs e as causas obtidas pelos revisores. Houve concordância de diagnósticos em apenas 13 casos de gastroenterite e dez de broncopneumonias, totalizando $15 \%$ do total de óbitos levados à necrópsia (em 12 outros óbitos, incluídos tanto pelos legistas quanto pelos revisores na categoria de "outros diagnósticos" na Tabela 8 , não houve concordância sobre a causa específica). Ressaltamos que, do total de 80 casos atestados como broncopneumonia pelos IMLs, 38 (48\%) foram julgados pelos revisores como devidos a morte súbita, oito $(10 \%)$ a gastroenterites e 24 $(30 \%)$ a outras causas que não as especificadas na Tabela 8. Deixou-se de registrar 19 casos de gastroenterite, e em outros 15 óbitos, por esta causa, o médico legista registrou a causa básica como sendo "desidratação"

TABELA 8

Causas básicas de óbitos infantis, declaradas pelos Institutos Médico-Legais (IMLs), comparados às causas estabelecidas pelos revisores. Região Metropolitana de Porto Alegre e Pelotas, RS, 1985.

\begin{tabular}{|c|c|c|c|}
\hline \multicolumn{2}{|c|}{ Causas básicas } & \multirow{2}{*}{ Número } & \multirow{2}{*}{$\%$} \\
\hline IMLs & Revisores & & \\
\hline Gastroenterite & Gastroenterite & 13 & 8 \\
\hline Gastroenterite & Morte súbita & 7 & 5 \\
\hline Gastroenterite & Outras & 8 & 5 \\
\hline Broncopneumonia & Broncopneumonia & 10 & 6 \\
\hline Broncopneumonia & Morte súbita & 38 & 24 \\
\hline Broncopneumonia & Outras (a) & 24 & 15 \\
\hline Broncopneumonia & Gastroenterite & 8 & 5 \\
\hline Outras (a) & Gastroenterite & 3 & 2 \\
\hline Outras (a) & Broncopneumonia & 1 & 1 \\
\hline Outras (a) & Morte súbita & 11 & 7 \\
\hline Outras (a) & Mal-definidas & 2 & 1 \\
\hline Outras (a) & Outras (a) & 12 & 8 \\
\hline Sem registro & Gastroenterite & 8 & 5 \\
\hline Sem registro & Broncopneumonia & 2 & 1 \\
\hline Sem registro & Morte súbita & 6 & 4 \\
\hline Sem registro & Mal-definida & 3 & 2 \\
\hline Tota! & & 156 & 99 \\
\hline
\end{tabular}

Obs.: (a) Ou tras causas não especificadas nesta Tabela. 


\section{DISCUSSÃO}

A metodologia utilizada permitiu detectar um sub-registro total de $8,3 \%$ dos óbitos estudados, concentrado principalmente na cidade de Pelotas, confirmando os achados de estudo anterior ${ }^{1}$. Este percentual de sub-registro chama a atenção, principalmente se levarmos em conta que apenas foram estudados óbitos pósperinatais ocorridos em áreas urbanas, e que o Rio Grande do Sul é considerado como um dos Estados com melhores estatísticas vitais.

Em termos de precisão da causa de óbito declarada nos atestados, chama a atenção o baixo índice de concordância observado em relação à causa estabelecida pela equipe da pesquisa. As distorções no preenchimento de atestados levam a uma subestimativa dos óbitos por gastroenterites e das mortes súbitas, e a uma sobreestimativa dos óbitos por broncopneumonia e por septicemia. Em particular, muitos óbitos por gastroenterite e por desnutrição foram atribuidos a septicemia, enquanto que mortes súbitas foram classificadas como broncopneumonias.

Como conseqüência, o número de mortes súbitas foi grosseiramente subestimado. Deve-se notar, no entanto, que a presente investigação de caráter retrospèctivo, sem dispor de apoio adequado em anatomia patológica, esteve sujeita a erros no diagnóstico desta síndrome, uma vez que foi utilizado um critério eminentemente clínico" ${ }^{11}$.

É também difícil avaliar o papel de desnutrição como causa básica de óbito em um estudo retrospectivo, uma vez que o peso obtido no. hospital por ocasião da admissão da criança já terá, muito provavelmente, sido afetado pelo episódio de doença. Além disso, a presença de desnutrição não pode ser avaliada em cerca de metade das crianças, devido à inexistência de registro de peso. Mesmo para as crianças que haviam sido pesadas, o critério para inclusão de desnutriçăo como causa básica foi arbitrário, escolhendo-se as crianças com um peso inferior à mediana da população de referência $\mathrm{NCHS}^{7}$, menos três desvios-padrão. Este critério é pouco mais sensivel - mas também menos específico - do que havia sido utilizado na Investigação Latinoamericana de Mortalidade na Infância (desnutrição de 3 . grau de Gomez ${ }^{3}$, ou seja, aproximadamente quatro desvios-padrão abaixo da mediana).

Uma das mais importantes consequiências dos erros na atribuição da causa de óbito foi a conclusão equivocada de que $o$ número de óbitos por infecções respiratórias era superior ao de óbitos por gastroenterites, quando o oposto era verdadeiro. Este achado tem importantes implicações em termos de programas de prevenção e controle de morbi-mortalidade infantil.

Outro dado preocupante foi a baixa qualidade dos atestados preenchidos por muitos dos médicos legistas. Maior colaboração entre os serviços de Medicina Legal e de Estatísticas Vitais é imprescindível.

$O$ estudo também confirmou o valor da 'necrópsia verbal', ou seja, de uma entrevista colhida por um médico junto aos pais das crianças falecidas e aos médicos que as assistiram. Em nossa avaliação, estes dados, suplementados pelas informações dos prontuários clínicos quando disponíveis, permitiram obter um grau adequado de certeza no estabelecimento da causa básica para cerca de $90 \%$ dos óbitos.

Em resumo, a qualidade global dos atestados de óbitos infantis nas áreas estudadas é insatisfatória. É imprescindivel salientar ao profissional médico, durante sua formação ou pela educação médica continuada, a importância do preenchimento adequado dos atestados de óbito para os programas de prevenção e controle da morbi-mortalidade infantil. Este parece ser mais um reflexo da má qualidade de grande parte dos serviços de saúde no Brasil. 
NOBRE, L.C. et al. [Assessment of the quality of information on basic causes of infant death in Rio Grande do Sul (Brazil)]. Rev. Saúde públ., S. Paulo, 23: 207-13, 1989.

ABSTRACT: The validity of the official information on the causes of infant deaths was studied in the Brazilian cities of Porto Alegre and Pelotas in 1985. Using data collected for a population-based casecontrol study of infant mortality due to infectious diseases or malnutrition, a comparison was made between the causes of death reported on the death certificates and those obtained after a careful review of case-notes and a medical interview with the parents of the deceased infants. Official death certificates showed an excess of deaths attribute to bronchopneumonia (ICD 485X) and septicemia (ICD 038.9), and an underestimation of the number of deaths due to diarrheal diseases (ICD 009.1) and of sudden infant deaths (ICD 798.0). The overall rate of agreement between official and revised certificates, in terms of groups of causes of death, was only $27.9 \%$. Lower respiratory infections, which were the leading infectious cause of infant deaths according to official statistics, were superseded by diarrheal diseases after this revision.

UNITERMS: Infant mortality. Diseases classification. Death certificates

\section{REFERÊNCIAS BIBLIOGRÁFICAS}

1. BARROS, F.C.; VICTORA, C.G.; TEIXEIRA, A.M.B.; PUERTO FILHO, M. Mortalidade perinatal e infantil em Pelotas, Rio Grande do Sul: nossas estatísticas são confiáveis? Cad. Saúde públ., 1:34858,1985 .

2. FLEISS, J.L. Statistical methods for rates and proportions. 2nd ed. New York, John Wiley \& Sons, 1981.

3. FONSECA, L.A. \& LAURENTI, R. A qualidade da certificação médıca da causa de morte em São Paulo. Rev. Saúde públ., S. Paulo, 8:21-9, 1984.

4. LAURENTI, R. Doenças respiratórias como causa de morte no Municipio de São Paulo, SP (Brasil). Rev. Saúde públ., S. Paulo, 15:353-63, 1981.

5. LAURENTI, R.; JORGE, M.H.P.; LEBRÃO, M.L.; GOTLIEB, S.L.D. Estatísticas de saude. São Paulo, EPU/EDUSP, 1985.

6. MANUAL da classificação estatística internacional de doenças, lesões e causas de óbito; Revisão 1975. São Paulo, Centro da OMS para Classificação de Doenças em Português, 1980. v. 1.

7. NATIONAL CENTER FOR HEALTH STATISTICS. NCHS growth charts, 1976. Mthly vital statist. Rep., 25(3): 1-22, 1976.

8. PUFFER, R.R. \& GRIFFITH, G.W. Características de la mortalidad urbana. Washington, D.C., Organizacion Panamericana de la Salud, 1968. (OPS - Publicacion cientifica, 151).

9. PUFFER, R.R. \& SERRANO, C.V. Patterns of mortality in childhood. Washington, D.C., Pan American Health Organization, 1973. (PAHO - Scientific Publication, 262).
10. SECRETARIA DA SAÚDE E DO MEIO AMBIENTE DO ESTADO. Estatísticas de saúde: mortalidade 1984. Porto Alegre, 1986.

11. VICTORA, C.G.; NOBRE, L.C.; LOMBARDI, C.; TEIXEIRA, A.M.B.; FUCHS, S.M.C.; MOREIRA, L.B.; GIGANTE, L.P.; BARROS, F.C. Quadro epidemiológico das mortes súbitas na infância em cidades gaúchas (Brasil), Rev. Saúde públ., S. Paulo, 21:490-6, 1987.

12. VICTORA, C.G.; SMITH, P.G.; VAUGHAN, J.P.; NOBRE, L.C.; LOMBARDI, C.; TEIXEIRA, A.M.B.; FUCHS, S.M.C.; MOREIRA, L.B.; GIGANTE, L.P.; BARROS, F.C. Evidence for a strong protective effect of breast-feeding against infant deaths due to infectious diseases in Brazil. Lancet, 2:319-22, 1987.

13. VICTORA, C.G.; SMITH, P.G.; VAUGHAN, J.P.; NOBRE, L.C.; LOMBARDI, C.; TEIXEIRA, A.M.B.; FUCHS, S.M.C.; MOREIRA, L.B.; GIGANTE, L.P.; BARROS, F.C. Influence of birthweight on infectious diseases mortality: a casecontrol study. Pediatrics, 81:807-11, 1988.

14. VICTORA, C.G.; SMITH, P.G.; VAUGHAN, J.P.; NOBRE, L.C.; LOMBARDI, C.; TEIXEIRA, A.M.B.; FUCHS, S.M.C.; MOREIRA, L.B.; GIGANTE, L.P.; BARROS, F.C. Infant mortality due to diarrhoea, related to the risk of water supply, sanitation and housing. Int. J. Epidem., 17(3); 1988 [no prelo].

Recebido para publicação em 23/9/88. Aprovado para publicação em 4/4/89. 